\title{
THE STUDY OF TIMING RELATIONSHIPS WHICH ARISE WHEN USING A TELEVISION CCD CAMERA WATEC WAT-902H2 SUPREME IN ASTRONOMICAL INVESTIGATIONS
}

\author{
V.V.Dragomiretsky, A.V.Ryabov, N.I.Koshkin \\ Astronomical Observatory, Odessa National University \\ Shevchenko Park, 65014, Odessa, Ukraine \\ andryabov(a)gmail.com
}

\begin{abstract}
The present paper describes the study of timing relationships which arise when using an analogue CCD camera Watec WAT-902H2 Sup in astronomical investigations, particularly in time-domain measurements of LEO satellites which are fast-moving against stellar background.
\end{abstract}

An analogue CCD camera Watec WAT-902H2 Sup is used to observe artificial Earth satellites using theodolite KT-50 at the Astronomical Observatory of Odessa National University. Specific character of the LEO satellite observations requires recording instants of observations with the highest possible precision (Flohrer, 2008; Shakun \& Koshkin, 2014).

To carry out the time reference to the UTC time scale, a timing signal from a GPS receiver is introduced into the CCD camera video output signal. The rising edge of the timing signal is synchronised with the beginning of a UTC second. The number of the UTC second is transmitted using a special code which allows of further automatic procession. The procedure of time referencing is described in detail in (Dragomiretsky et al., 2013; Research Report No 365, 2008).
As the reference to timing signals is carried out in the video output, there is a certain time interval between the instant of an astronomical event's observation and the instant of time referencing. To determine the length of this time interval the laboratory tests of the CCD camera Watec WAT-902H2 Sup were performed.

At the first stage of the laboratory tests the CCD camera was exposed to the strobe illumination with the further video signal recording and processing. The strobe flash duration was $1 \cdot 10^{-6} \mathrm{~s}$ with the frequency of $20-100 \mathrm{~Hz}$. The indicated tests were carried out both using uniform strobe illumination of the whole sensitive surface and with the light source focusing on a given area of the sensitive surface. Based on the results of these two tests, we concluded that when the CCD camera WAT-902H2 Sup runs in the mode of low ambient illumination with disabled AGC system, simultaneous recording of all events on the whole sensitive surface is performed. At that it is only possible to discuss the instants of the beginning and end of exposure in a similar way to the application of photosensitive emulsions.

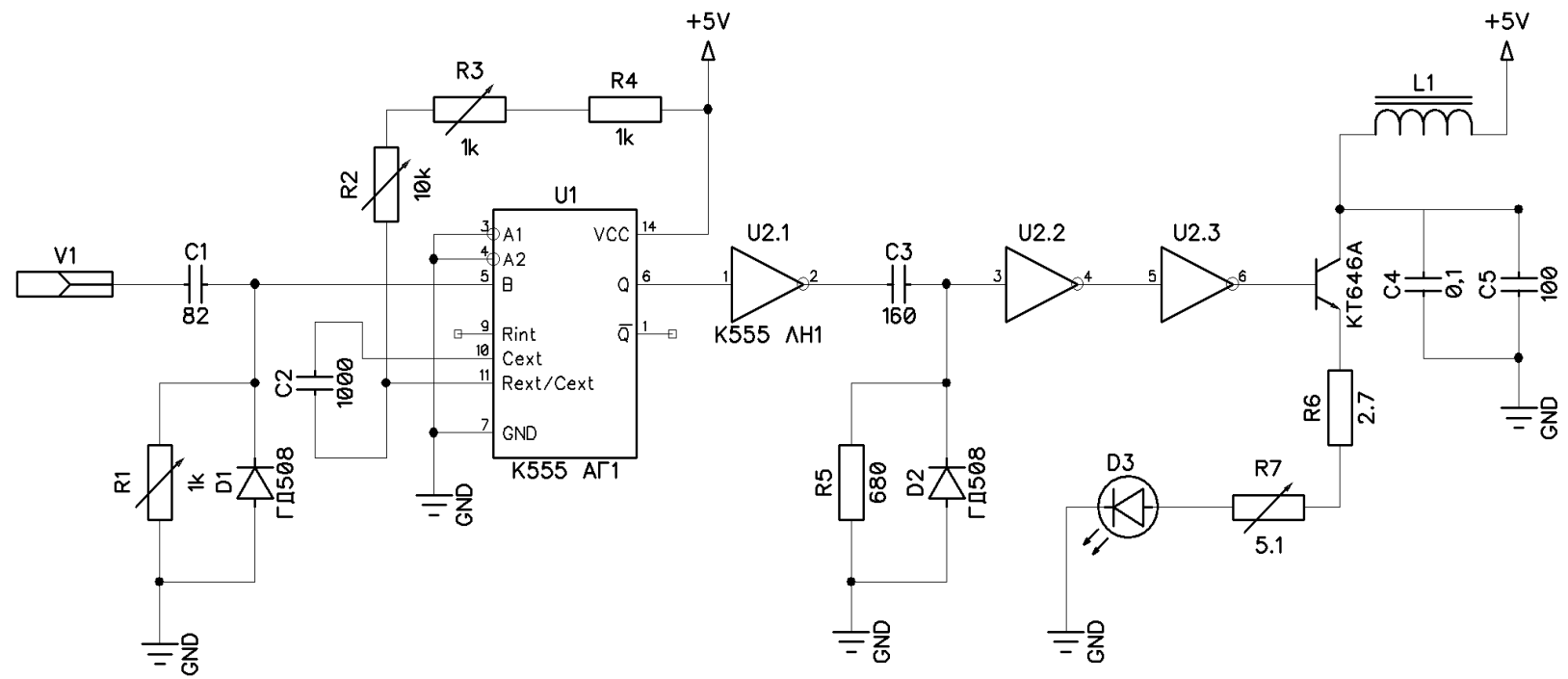

Figure 1. 
Further investigations are focused on the determination of time intervals between the instant of recording of an astronomical event and the instant of time referencing of the video output with GPS signals. We used a stroboscope synchronised with procedure signals generated by the CCD camera. The stroboscope circuit is presented in Fig. 1. The strobe is synchronised with the signal $V 1$ generated by the timing controller CXD2463R integrated into the CCD camera (http://www.alldatasheet.com/datasheetpdf/pdf/47009/SONY/CXD2463R.html). The connection of a stroboscope did not impair the CCD camera performance. The stroboscope circuit produces an intense electric impulse of $0.4 \mu$ s duration with a delay of $1 \div 8 \mu$ s relative to the rising edge of the signal $V 1$. This delay is controlled by the resistors $R 2$ and $R 3$. The stable synchronisation level is set by the resistor $R 1$. The light pulse level is controlled by the resistor $R 7$. It is assumed that the duration of the LED afterglow emission does not exceed $0.5 \mu \mathrm{s}$. Thus, the light pulse duration is no more than $1 \mu \mathrm{s}$. The CCD camera's dead zone (Fig. 2) was found by changing the light pulse delay. The dead zone where the sensitivity is completely lost is between the falling edge of the signal $V 1$ and the rising edge of the signal V3 (http://www.alldatasheet.com/datasheet-pdf/pdf/ 47009/SONY/CXD2463R.html). The zone of reduced sensitivity was also found in the range between the rising edge of the signal $V 1$ and the falling edge of the signal $V 3$.

It can be concluded from the above that the exposure of the previous frame ends when the signal $V 1$ is active while the exposure of the next frame begins when the signal $V 3$ is active.
The shape of the CCD camera video signal output is shown in Fig. 3. The beginning of the $\mathrm{X}$-axis corresponds to the rising edge of the signal VI. In fact, a part of the video output signal between the rising edge of the signal $V 1$ and the beginning of the first image line corresponds to a certain part of the vertical synchronization pulse (https://commons.wikimedia.org/wiki/File:BAS_Zeilensig nal_unmoduliert_1.svg). As this part of the frame sync pulse remains constant when the camera WAT-902H2 Sup is operated in the above-indicated mode, it is possible to calculate the time constant correction between the end of the previous frame's exposure and the beginning of the first whole line of the corresponding video output signal.

As follows from the graph presented in Fig. 3, the time interval between the instant of time corresponding to the rising edge of the signal $V 1$ and the instant corresponding to the beginning of the first whole line of the video output signal is equal to $24 \mu \mathrm{s}$ and nine complete periods corresponding to the video output line duration. Thus, the indicated time interval is $\Delta t=24+9.64=600(\mu s)$. As follows from the graph presented in Fig. 2, the middle of the dead zone where the sensitivity is lost is separated in time by $3.55 \mu$ s from the instant of the $V 1$ signal's rising edge. Therefore, the time interval between the instant of the end of the previous frame's exposure and the instant of the beginning of the first whole data line of the corresponding video output signal is $t=(\Delta t-2.3) \mu s$, which makes $597.7 \mu$ s.

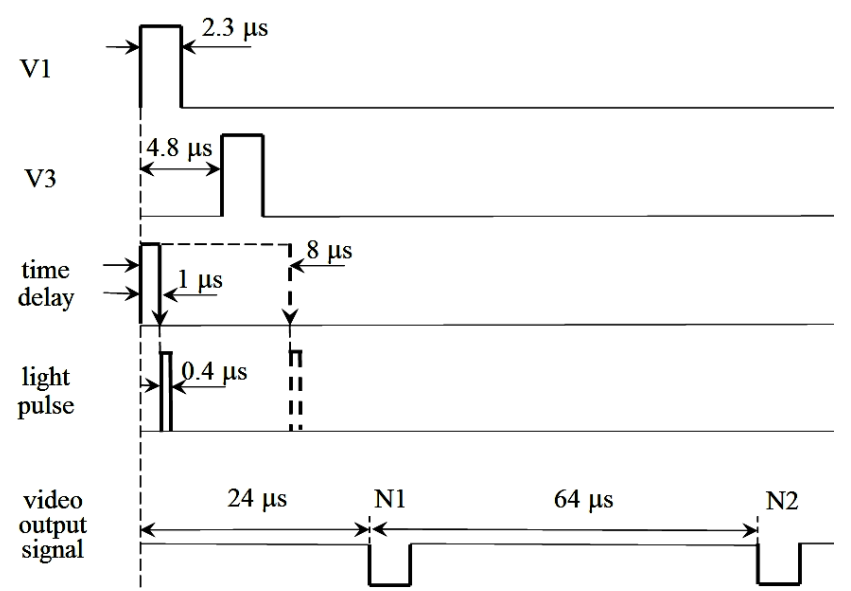

Figure 2. 


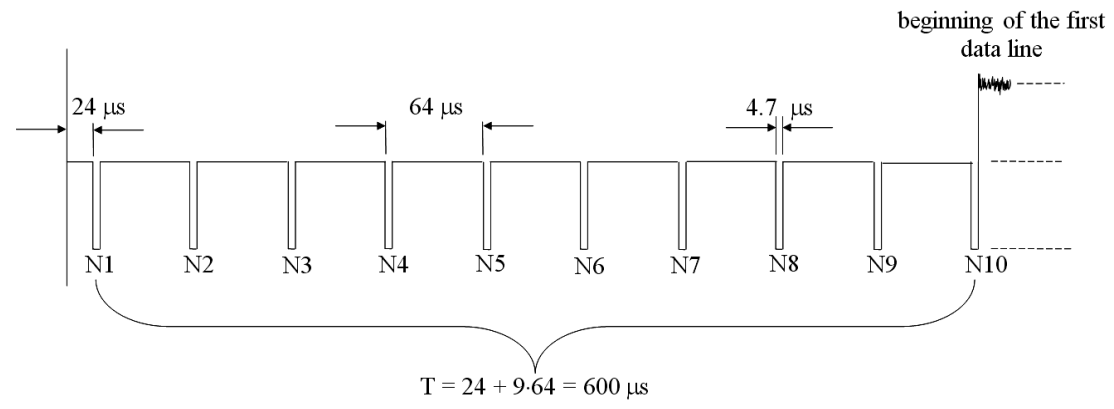

Figure 3.

Accounting for the duration of the detected dead zone, the frame exposure time for the indicated mode is 19.9975 $m s$. Thus, the time interval between the instant of the beginning of the previous frame's exposure and the instant of the beginning of the first whole data line of the corresponding video output signal is $(t+19.9975) \mathrm{ms}$, which makes $20.5952 \mathrm{~ms}$. If the frame exposure is reduced to the average instant of time, then the time interval between the average instant of the previous frame's exposure and the instant of the beginning of the first whole data line of the corresponding video output signal is $t+19.9976 / 2(\mathrm{~ms})$, which makes $10.59645 \mathrm{~ms}$.

The obtained time corrections are used to specify the instants of observations of artificial Earth satellites using theodolite KT-50. The video signal standards given in https://commons.wikimedia.org/wiki/File:BAS_Zeilensig nal_unmoduliert_1.svg were used to perform calculations. The time interval between the instant of the beginning of the first whole data line and the instant of the arrival of a timing signal from a GPS receiver is determined according to the methods specified in (Dragomiretsky et al., 2013; Research Report No 484, 2014].

\section{References}

Flohrer C. Mutual Validation of Satellite-Geodetic Techniques and Its Impact on GNSS Orbit Modeling. Volume 75. Swiss Geodetic Commission, Zurich, Switzerland, 2008. (http://www.sgc.ethz.ch/publications.html)
Shakun L.S., Koshkin N.I. Determination of Visible Coordinates of the Low-Orbit Space Objects and Their Photometry by the CCD Camera with the Analogue Output. Initial Image Processing. Adv. in Space Res., 53, Issue 12, 2014, 1834-1847.

Dragomiretsky V.V., Koshkin N.I., Korobeinikova E.A., Melikyants C.M., Ryabov A.V., Strakhova S.L., Terpan S.S., Shakun L.S. Timing of Satellite Observations for Telescope with TV CCD Camera. Bulletin of Ukrainian Earth Orientation Parameters Laboratory, 2013, 8, 75-78.

Research Report No 365: Investigation of Drag-Free Motion of Artificial Earth Satellites and Asteroids as well as Ecological Problems in Near-Earth Space. Odessa, ONU, 2008, 111p. (Registration Number 0106U001670).

http://www.alldatasheet.com/datasheet-pdf/pdf/47009/ SONY/CXD2463R.html

Video Signal Standards: BAS-Zeilensignal und Verzögertes Signal (https://commons.wikimedia.org/ wiki/File:BAS_Zeilensignal_unmoduliert_1.svg)

Research Report No 484: The Study of Motion and Fragmentation of Meteor and Artificial Bodies in the Earth's Atmosphere and Interplanetary Space. Odessa, ONU, 2014, 167p. (Registration Number 0112U001749). 